\section{$C_{\text {cituris }}$}

epartment of Radiology, Barnet Hospital, Royal Free London NHS Foundation Trust, London, UK

${ }^{2}$ Department of Respiratory Medicine, Barnet Hospital, Royal Free London NHS Foundation Trust, London, UK

\section{Correspondence to} Dr Aniket N Tavare, Department of Radiology, Barnet Hospital, Royal Free London NHS Foundation Trust, Wellhouse Lane, London EN5 3DJ, UK; aniket.tavare@gmail.com

Received 21 March 2017 Revised 28 April 2017

Accepted 15 May 2017 Published Online First 25 July 2017

\title{
Pneumomediastinum and pneumorrhachis from recreational nitrous oxide inhalation: no laughing matter
}

\author{
Aniket N Tavare, ${ }^{1}$ Dana Li, ${ }^{1}$ Samanjit S Hare, ${ }^{2}$ Dean D Creer ${ }^{1}$
}

A 16-year-old girl with no medical history presented to our emergency department with rapid onset and progressive facial and neck swelling, dyspnoea and dysphonia. The previous night she had attended a nightclub, had sniffed ketamine and inhaled nitrous oxide. On examination she had swelling of her face, neck and upper torso with palpable crepitus. Scattered bilateral expiratory wheeze was present on auscultation. She was mildly tachycardic and tachypnoeic but other observations were normal. Neurological examination was normal. A chest radiograph demonstrated extensive subcutaneous emphysema. CT scan of the neck, chest and abdomen without intravenous contrast confirmed the presence of extensive subcutaneous gas within the face, neck and thorax, including pneumomediastinum, pneumopericardium and small bilateral pneumothoraces (figure 1). Air was also seen tracking within the spinal canal in the cervical and thoracic regions, a phenomenon known as pneumorrhachis (figure 1, arrow).

She later reported that she had taken the nitrous oxide directly from the pressurised canister (figure 2). We felt this was likely to account for the disproportionate volume of subcutaneous and mediastinal gas. Although a pharyngeal site of perforation was thought most likely, given the distribution of subcutaneous gas and presumed localised barotrauma-induced mechanism, flexible nasendoscopy was normal.

The diffuse subcutaneous emphysema, including pneumorrhachis, was managed conservatively with supplemental oxygen therapy.

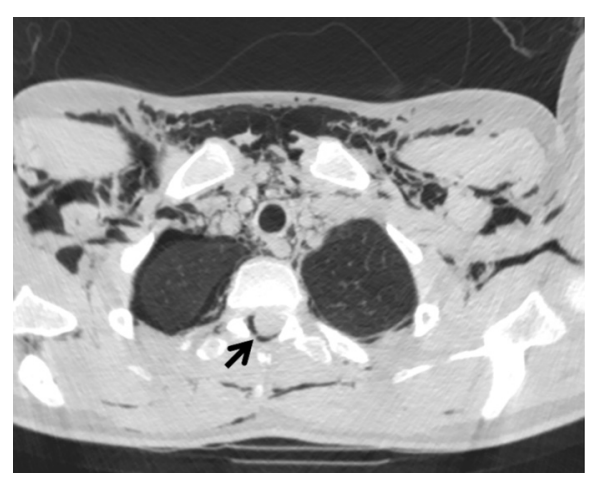

Figure 1 Axial CT of the upper thorax showing extensive subcutaneous and mediastinal gas, small bilateral pneumothoraces along with pneumorrhachis (arrow).

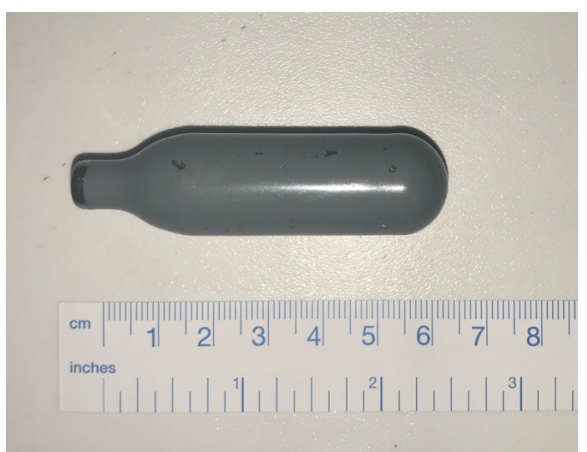

Figure 2 Typical pressurised canister in which nitrous oxide is sold for commercial and recreational use.

There was progressive resolution over the next few days and the patient was well when reviewed at 1 month with a normal chest radiograph.

Pneumorrhachis is an unusual phenomenon ${ }^{1}$ which is usually asymptomatic and is invariably accompanied by extra-anatomic gas elsewhere. The gas may lie within or outside the dura, depending on the underlying pathophysiology. Both traumatic and non-traumatic causes have been reported, including iatrogenic aetiologies and following inhalation of illicit substances. ${ }^{2}$ There is little consensus regarding management. Obtaining specialty reviews (eg, otorhinolaryngology, gastroenterology and neurosurgery) is prudent to attempt to find the source of breach and guide further investigations and management.

Nitrous oxide is widely used as both an inhalation anaesthetic agent and a recreational drug. While medical use is falling, recreational use is increasing, with $7.6 \%$ of persons aged 16-24 years having reported use in the last year, ${ }^{3}$ commonly at parties and festivals. A widely used method for recreational consumption involves decanting the contents of the pressurised canister into a balloon, presumably to allow controlled administration in small aliquots. The typical canister in figure 2 contains $8 \mathrm{~g}$ of nitrous oxide, equivalent to $8 \mathrm{~L}$ at standard temperature and pressure. We hypothesise that our patient was experiencing the dissociative effects of the ketamine and therefore was insensate to the localised barotrauma from the pressurised flow from the pressurised canister.

Contributors ANT wrote the manuscript and prepared the images. DL edited the manuscript and cared for the patient. SSH 
edited the manuscript and images. DDC edited the manuscript and was clinician responsible for the patient.

Competing interests None declared.

\section{Patient consent Obtained.}

Provenance and peer review Not commissioned; externally peer reviewed.

(c) Article author(s) (or their employer(s) unless otherwise stated in the text of the article) 2018. All rights reserved. No commercial use is permitted unless otherwise expressly granted.

\section{REFERENCES}

1 Oertel MF, Korinth MC, Reinges MH, et al. Pathogenesis, diagnosis and management of pneumorrhachis. Eur Spine J 2006;15(Suppl 5):636-43.

2 Bernaerts A, Verniest T, Vanhoenacker $F$, et al. Pneumomediastinum and epidural pneumatosis after inhalation of "Ectasy". Eur Radiol 2003;13:642-3.

3 Randhawa G, Bodenham A. The increasing recreational use of nitrous oxide: history revisited. Br J Anaesth 2016;116:321-4. 